\title{
MIMO OTA Testing Based on Transmit Signal Processing
}

\author{
Jesús Gutiérrez, Jesús Ibáñez, and Jesús Pérez \\ Department of Communications Engineering, University of Cantabria, 39140 Santander, Spain \\ Correspondence should be addressed to Jesús Pérez; jperez@gtas.dicom.unican.es
}

Received 1 February 2013; Revised 17 May 2013; Accepted 19 May 2013

Academic Editor: Pablo Padilla

Copyright (C) 2013 Jesús Gutiérrez et al. This is an open access article distributed under the Creative Commons Attribution License, which permits unrestricted use, distribution, and reproduction in any medium, provided the original work is properly cited.

\begin{abstract}
Usually, multiple-input-multiple-output (MIMO) testbeds are combined with channel emulators for testing devices and algorithms under controlled channel conditions. In this work, we propose a simple methodology that allows over-the-air (OTA) MIMO testing using a MIMO testbed solely, avoiding the use of channel emulators. The MIMO channel is emulated by linearly combining the signals at the testbed transmitter. The method is fully flexible, so it is able to emulate any equivalent baseband narrowband MIMO channel by adequately selecting the weights of the linear combination. We derive closed-form expressions for the computation of such weights. To prove its feasibility, the method has been implemented and tested over a commercial MIMO testbed.
\end{abstract}

\section{Introduction}

Multiple-input-multiple-output (MIMO) testbeds [1-3] allow over-the-air (OTA) testing of MIMO devices and algorithms in the laboratory. However, the channels in these indoor scenarios can be quite different from the channels where MIMO systems operate. So, MIMO testbeds are usually combined with MIMO channel emulators for carrying out the tests under desired and controlled channel conditions. However, channel emulators result in an increase in equipment cost. Some OTA emulation methods combine conventional channel emulators with anechoic chambers to fully control the propagation environment [4-6]. These solutions are fully flexible but expensive.

On the other hand, OTA channel emulation can be performed using reverberation chambers where the propagation environment is physically modified to get the desired channel response. Reverberation chambers can work either standing alone [7], or combined with conventional channel emulators $[8,9]$. Their main drawbacks are the chamber cost and the lack of flexibility to emulate specific channel realizations. Both methods, based on anechoic and reverberation chambers, are used in combination with a MIMO testbed for MIMO testing.

In this work, we propose a methodology that allows narrowband OTA MIMO testing using the MIMO testbed solely, avoiding the use of channel emulators and additional facilities. It is based on the fact that the MIMO channel encountered in indoor research laboratories can be considered time invariant when the environment is static [10]. In a preliminary stage, this channel must be estimated by the MIMO testbed [10], including the device under test (DUT). Then, during the testing stage, the signals at the transmitter $(\mathrm{Tx})$ are linearly combined in order to emulate the desired MIMO channel. This operation can be easily performed at the Tx baseband (BB) processor. The DUT(s) can be at the Tx and/or at the receiver (Rx). The DUT can be also a complete $\mathrm{Rx}$ able to estimate the channel. The proposed method is also well suited to test MIMO-specific algorithms (space-time coding, spatial multiplexing, etc.), that are usually implemented in the $\mathrm{BB}$ processors. The proposed method emulates baseband equivalent channel response, so, unlike reverberation chambers, it cannot emulate separately the effects of the multipath propagation scenario, antennas, or other embedded elements.

We consider two types of channel emulation: deterministic and stochastic. In the first case, the goal is to emulate a given channel realization (or a given sequence of channel realizations). We show closed-form expressions for the coefficients of the linear combination as functions of the actual channel in the laboratory and of the channel to emulate. In stochastic channel emulation, the objective is just to emulate 


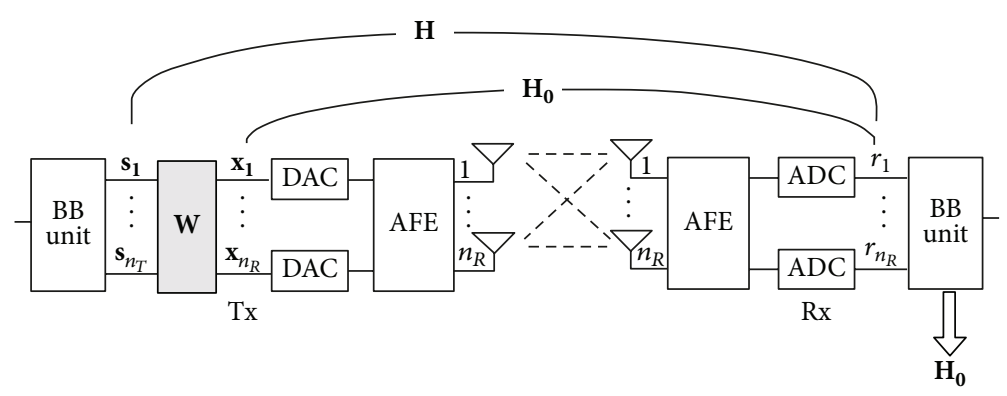

FIGURE 1: Proposed scheme using linear signal processing at the Tx. AFE denotes the analog front end, BB denotes baseband, and the DACs and ADCs are the digital-to-analog and the analog-to-digital converters, respectively.

a channel as a stochastic process with desired statistics (mean, spatial autocovariance, etc.), regardless of the channel realizations. The stochastic emulation is motivated by the fact that the field programmable gate arrays (FPGA) or digital signal processors (DSP) of the Tx testbeds can efficiently generate realizations of multiple independent and identically distributed (i.i.d.) random variables. Adequate linear combinations of such variables can be used to generate the coefficients for the channel emulation with desired statistics.

The proposed method has been implemented and tested over a commercial MIMO testbed in our laboratory. The experimental results show the accuracy of the method for OTA MIMO testing in any channel condition.

The rest of the paper is organized as follows. Section 2 describes the proposed scheme. From this, we derive closedform expressions for the weights as a function of the channel to emulate and the actual channel in the laboratory. Section 3 deals with the implementation in a commercial testbed. Section 4 shows experimental results that validate the method. Finally, Section 5 is devoted to the concluding remarks.

\section{MIMO Channel Emulation Scheme}

Figure 1 depicts the proposed scheme for MIMO testing and channel emulation. To emulate a MIMO channel with $n_{T}$ transmit and $n_{R}$ receive antennas, a testbed Tx with $n_{R}$ antennas is required. Let $\mathbf{H}_{0} \in \mathbb{C}^{n_{R} \times n_{R}}$ be the equivalent baseband channel response between the testbed Tx and the $\mathrm{Rx}$ for a specific location in the laboratory, including the DUT (or the DUTs). As it was mentioned, the DUTs can be at the Tx and/or Rx. The DUT could be even a complete Rx. In any case, the $\mathrm{BB}$ processor at the Rx must estimate the channel, $\mathbf{H}_{0}$, which is assumed to be constant during the process. Let $\mathbf{s} \in \mathbb{C}^{n_{T} \times 1}$ be a vector comprising the equivalent baseband input signals. They are linearly combined by a weights matrix $\mathbf{W} \in \mathbb{C}^{n_{R} \times n_{T}}$ at the testbed $\mathrm{Tx}$ to produce the signal vector $\mathbf{x} \in \mathbb{C}^{n_{R} \times 1}$,

$$
\mathbf{x}=\mathbf{W} \mathbf{s} \text {. }
$$

Then, the testbed transmits $\mathbf{x}$. In the absence of noise, the receive signal vector will be

$$
\mathbf{r}=\mathbf{H}_{0} \mathbf{x}=\mathbf{H}_{0} \mathbf{W} .
$$

Therefore, the baseband equivalent channel

$$
\mathbf{H}=\mathbf{H}_{0} \mathbf{W} \text {. }
$$

2.1. Deterministic Channel Emulation. According to (3), the weights matrix to emulate a given channel realization, $\mathbf{H}$, will be

$$
\mathbf{W}=\left(\mathbf{H}_{0}\right)^{-1} \mathbf{H}
$$

where we assume that $\mathbf{H}_{0}$ has been previously estimated by the $\mathrm{BB}$ unit at the $\mathrm{Rx}[10]$. In (4), we assume that $\mathbf{H}_{0}$ is invertible. If this was not the case, we can change the location of the testbed in the laboratory to get a well-conditioned $\mathbf{H}_{0}$.

To emulate a given sequence of channel realizations (possibly time correlated), $\mathbf{H}[n]$, we will obtain the corresponding sequences of weights, $\mathbf{W}[n]$, from (4), assuming that $\mathbf{H}_{0}$ is time invariant. Otherwise, we should reestimate $\mathbf{H}_{0}$ and repeat the process whenever it changes. Note that the emulation of time-correlated channel sequences is straightforward.

In general, the emulation of a $n_{R} \times n_{T}$ MIMO channel requires a testbed Tx with $n_{R}$ antennas. Therefore, to emulate a single-input-multiple-output (SIMO) channel with $n_{R}$ antennas, a testbed Tx with $n_{R}$ antennas is still required. But for multiple-input-single-output (MISO) channel emulation, $n_{R}=1$, so we only need a single antenna testbed Tx. Obviously, this simple scheme is valid when the DUT is at the Rx or it is the complete Rx. Otherwise, if the DUT is at the radiofrequency part of the Tx, we still need a Tx with $n_{T}$ branches.

2.2. Stochastic Channel Emulation. Now, the channel to emulate, $\mathbf{H}$, is a random process with a given distribution and spatial covariance. Using our scheme, the statistics of $\mathbf{H}$ can be emulated by using a random weights matrix $\mathbf{W}$ with adequate distribution and spatial covariance. In the following lines, we will derive the statistical properties of $\mathbf{W}$ as a function of the required statistics for $\mathbf{H}$, assuming that $\mathbf{H}_{0}$ is time invariant (deterministic) during the emulation process. We distinguish two cases regarding the mean of $\mathbf{H}$ : emulation of MIMO channels with zero mean and with arbitrary mean.

2.2.1. Emulation of Channels with Zero-Mean. Let $\mathbf{H}$ be a random channel matrix with zero mean, $E[\mathbf{H}]=\mathbf{0}$, and 
correlation matrix R. From (3), W must be also zero-mean, and $E[\mathbf{W}]=\mathbf{0}$. The channel correlation matrix is defined as

$$
\mathbf{R}=E\left[\operatorname{vec}(\mathbf{H}) \operatorname{vec}(\mathbf{H})^{H}\right]
$$

where $\operatorname{vec}(\mathbf{A})$ is the operator that stacks matrix $\mathbf{A}$ into a vector columnwise. From (3),

$$
\operatorname{vec}(\mathbf{H})=\operatorname{vec}\left(\mathbf{H}_{0} \mathbf{W}\right)=\left(\mathbf{I}_{n_{T}} \otimes \mathbf{H}_{0}\right) \operatorname{vec}(\mathbf{W})
$$

where $\otimes$ denotes the Kronecker product. Combining (5) and (6),

$$
\mathbf{R}=\left(\mathbf{I}_{n_{T}} \otimes \mathbf{H}_{0}\right) \mathbf{R}_{W}\left(\mathbf{I}_{n_{T}} \otimes \mathbf{H}_{0}^{H}\right)
$$

where $\mathbf{R}_{W}=E\left[\operatorname{vec}(\mathbf{W}) \operatorname{vec}(\mathbf{W})^{H}\right]$ is the correlation matrix of $\mathbf{W}$. Therefore, to get $\mathbf{R}$, the weights correlation matrix must be

$$
\mathbf{R}_{W}=\left(\mathbf{I}_{n_{T}} \otimes \mathbf{H}_{0}^{-1}\right) \mathbf{R}\left(\mathbf{I}_{n_{T}} \otimes\left(\mathbf{H}_{0}^{-1}\right)^{H}\right) .
$$

As example, to emulate a Rayleigh channel with correlation matrix $\mathbf{R}$, we will generate realizations of the matrix $\mathbf{W}$ according to

$$
\operatorname{vec}(\mathbf{W})=\mathbf{R}_{W}^{1 / 2} \operatorname{vec}\left(\mathbf{W}_{W}\right),
$$

where $\mathbf{R}_{W}$ is given by (8) and $\operatorname{vec}\left(\mathbf{W}_{W}\right)$ is an i.i.d. complex Gaussian vector: $\operatorname{vec}\left(\mathbf{W}_{W}\right) \sim \mathscr{C} \mathscr{N}\left(\mathbf{0}, \mathbf{I}_{n_{R} n_{T}}\right)$. The realizations of $\mathbf{W}_{W}$ can be efficiently generated, in real time, within the FPGA or DSP of the Tx.

2.2.2. Emulation of Channels with Arbitrary Mean. Let $\mathbf{H}$ be a random channel matrix with mean $E[\mathbf{H}]=\mathbf{M}$. From (3), $\mathbf{M}=\mathbf{H}_{0} \mathbf{M}_{W}$, where $\mathbf{M}_{W}=E[\mathbf{W}]$. Therefore, to emulate a channel with mean $\mathbf{M}$, the mean of the weights matrix must be

$$
\mathbf{M}_{W}=\mathbf{H}_{0}^{-1} \mathbf{M}
$$

Let us write that $\mathbf{H}=\widetilde{\mathbf{H}}+\mathbf{M}$ and $\mathbf{W}=\widetilde{\mathbf{W}}+\mathbf{M}_{W}$, where $\widetilde{\mathbf{H}}=\mathbf{H}_{0} \widetilde{\mathbf{W}}$. The covariance matrix of $\mathbf{H}, \mathbf{C}$, is the correlation matrix of $\widetilde{\mathbf{H}}$. Similarly, the covariance matrix of $\mathbf{W}, \mathbf{C}_{W}$, is the correlation matrix of $\widetilde{\mathbf{W}}$. Then, from (7),

$$
\mathbf{C}=\left(\mathbf{I}_{n_{T}} \otimes \mathbf{H}_{0}\right) \mathbf{C}_{W}\left(\mathbf{I}_{n_{T}} \otimes \mathbf{H}_{0}^{H}\right) .
$$

Therefore, to emulate a MIMO channel with covariance matrix $\mathbf{C}$, the covariance matrix of the coefficients must be

$$
\mathbf{C}_{W}=\left(\mathbf{I}_{n_{T}} \otimes \mathbf{H}_{0}^{-1}\right) \mathbf{C}\left(\mathbf{I}_{n_{T}} \otimes\left(\mathbf{H}_{0}^{-1}\right)^{H}\right) .
$$

As example, to emulate a Ricean channel with mean $\mathbf{M}$ and covariance matrix $\mathbf{C}$, we will generate realizations of $\mathbf{W}$ according to

$$
\operatorname{vec}(\mathbf{W})=\operatorname{vec}\left(\mathbf{M}_{W}\right)+\mathbf{C}_{W}^{1 / 2} \operatorname{vec}\left(\mathbf{W}_{W}\right),
$$

where $\mathbf{M}_{W}$ and $\mathbf{C}_{W}$ are given by (10) and (12), respectively. As it was mentioned, realizations of $\mathbf{W}_{W}$ can be easily generated, in real time, within the FPGA or DSP of the testbed Tx.

2.3. Emulation of MIMO Channels Based on Dual-Polarized Antennas. The use of dual-polarized antennas at the Tx and $\mathrm{Rx}$ leads to a $2 \times 2 \mathrm{MIMO}$ channel. The diagonal elements of the channel matrix correspond to transmission and reception on the same polarization, while off-diagonal elements correspond to transmission and reception on orthogonal polarization. Assuming Rayleigh fading, the channel is usually modeled approximately as follows [11-13]:

$$
\operatorname{vec}(\mathbf{H})=\operatorname{vec}(\mathbf{X}) \odot\left(\mathbf{R}^{1 / 2} \operatorname{vec}\left(\mathbf{H}_{\omega}\right)\right), \quad \mathbf{H}_{\omega} \sim \mathscr{C} \mathcal{N}(\mathbf{0}, \mathbf{I})
$$

where $\odot$ stands for the Hadamard product, $\mathbf{R}$ is now the socalled polarization correlation matrix (usually it is approximated in terms of the transmit and receive polarization correlation matrices: $\mathbf{R}=\mathbf{R}_{t}^{T} \otimes \mathbf{R}_{r}$ ), and $\mathbf{X}$ is the polarization leakage matrix given by

$$
\mathbf{X}=\left[\begin{array}{cc}
\sqrt{1-\alpha} & \sqrt{\alpha} \\
\sqrt{\alpha} & \sqrt{1-\alpha}
\end{array}\right] \text {, }
$$

where $\alpha$ is a parameter which depends on the cross polarization discrimination (XPD) of the antennas and of the cross polarization coupling (XPC) of the propagation environment, often collectively referred to as XPD.

In order to emulate a Rayleigh channel with given polarization correlation matrix, $\mathbf{R}$, and polarization leakage matrix, $\mathbf{X}$, one simply should use the weights vector $\mathbf{W}^{\prime}$ given by

$$
\mathbf{W}^{\prime}=\mathbf{X} \odot \mathbf{W}
$$

where $\mathbf{W}$ is obtained from (8) and (9) as a function of $\mathbf{R}$. Now, the emulated channel will be

$$
\mathbf{H}=\mathbf{H}_{0} \mathbf{W}^{\prime}=\mathbf{X} \odot \mathbf{H}_{0} \mathbf{W} .
$$

Then,

$$
\operatorname{vec}(\mathbf{H})=\operatorname{vec}(\mathbf{X}) \odot\left(\mathbf{I}_{n_{T}} \otimes \mathbf{H}_{0}\right) \operatorname{vec}(\mathbf{W})
$$

Considering (9) and (7),

$$
\begin{aligned}
\operatorname{vec}(\mathbf{H}) & =\operatorname{vec}(\mathbf{X}) \odot\left(\mathbf{I}_{n_{T}} \otimes \mathbf{H}_{0}\right) \mathbf{R}_{W}^{1 / 2} \operatorname{vec}\left(\mathbf{W}_{W}\right) \\
& =\operatorname{vec}(\mathbf{X}) \odot \mathbf{R}^{1 / 2} \operatorname{vec}\left(\mathbf{W}_{W}\right),
\end{aligned}
$$

which is exactly the model (14). 


\section{Hardware Implementation}

The requirements for the hardware implementation of the proposed MIMO OTA testing method are as follows.

(i) A Tx and an Rx with adequate number of antennas (see Section 2).

(ii) A weighting block at the Tx where the baseband signals, s, are linearly combined according to the adequate weights, $\mathbf{W}$.

(iii) Channel estimation capability at the $\mathrm{Rx}$ baseband processor.

The DUTs can be any part of the Tx and/or Rx (antennas, elements of the analog front ends (AFEs), MIMO algorithms implemented at the $\mathrm{BB}$ processors, etc.). The DUT can be also the complete $\mathrm{Rx}$, provided that its $\mathrm{BB}$ processor is able to estimate the equivalent baseband channel, $\mathbf{H}_{0}$.

Figure 2 shows the hardware implementation scheme.

3.1. Hardware Description. Regarding hardware implementation, in the Tx, we add a new block called weighting block to the existing BB processor. This block is in charge of applying the weights calculated in Section 2. In general, the weighting block consists of $n_{R} n_{T}$ complex multipliers (each one comprising 4 real multipliers and 2 real adders) and 2 complex adders (each one comprises 2 real adders). The baseband inphase and quadrature (IQ) signal samples, $\mathbf{s}$, are generated by the baseband $(\mathrm{BB})$ processor and weights, $\mathbf{W}$, are fed, along with the signal, to the complex multipliers. The outputs of the weighting block, $\mathbf{x}$, are converted to the analog domain by $n_{R}$ dual digital-to-analog converters (DAC) and then upconverted to RF signals by the analog front-end (AFE). As stated before, there does not exist additional processing or extra hardware at the receive side.

This methodology requires a preliminary estimation of the actual MIMO channel, $\mathbf{H}_{0}$, which is typically performed by the $\mathrm{Rx}$ processor at the $\mathrm{Rx}$ (it can be carried out as described in [10]). The weights are scaled within the range $[-1,1]$ to ensure that in their maximum value the IQ signals are not affected by saturation that can occur at the weighting block or at the DACs. If a higher dynamic range is desired, there exists a control that allows varying the transmit power amplifier (PA) gain at the AFE.

As Figure 2 shows, the weighting block is fitted into the transmit FPGA. The DACs have 14 bits of resolution, so the output of the weighting block must be truncated to 14 bits. The transmit weight vector falls in the range $[-1,1]$ with one bit for the sign and 15 bits for the decimal part.

3.2. System Operation. The whole system is able to work in two modes, each suited to different requirements. On the first mode, the system emulates a block-fading channel, that is, within a block-fading period, the desired channel, $\mathbf{H}$, remains constant, varying from block to block according to some underlying distribution. To emulate a block-fading channel, the user should change the weights from time to time, and as a result, the time among channel realizations is determined by user decisions and the delays related to weight uploading
TABLE 1: Average SNR of the estimates of $\mathbf{H}_{0}$.

\begin{tabular}{lcccc}
\hline & $h_{11}$ & $h_{21}$ & $h_{12}$ & $h_{22}$ \\
\hline SNR (dB) & 28.8073 & 22.3865 & 22.5993 & 21.7302 \\
\hline
\end{tabular}

and application. This implementation takes advantage of the fact that the maximal Doppler frequency of typical fading channels is often much smaller than the symbol rate or even the data frame size and that fading variation within channel coherence time is small.

The second operation mode considers a sample-based weight application, hence the possibility of emulating high demand variability environments. In this case, the weight realizations obtained from (4) and the transmit signals are stored in the synchronous dynamic random access memory (SDRAM) and are applied in a sample by sample manner. In a sample-based weight delivering, the sampling frequency of the transmitter determines the time resolution at which the channel realizations are applied. The limitation is that the data must be prestored in memory, which is finite.

\section{Validation and Test}

The method described in the previous section was implemented in a $2 \times 2$ testbed (see Figure 2), operating at $5.6 \mathrm{GHz}$, using the multiantenna testbed nodes described in $[1,10]$. The aim was to emulate a sequence of 200 MIMO $2 \times 2$ channel realizations, $\mathbf{H}[n]$. The first 20 samples of $\mathbf{H}[n]$ are depicted in Figure 4 with circle markers.

Firstly, the actual channel $\mathbf{H}_{0}$ between the Tx and $\mathrm{Rx}$ units of the testbed was estimated 100 times along 90 seconds using the method described in [10]. The estimates are depicted in Figure 3 showing the lack of variability of the channel in the laboratory.

Table 1 illustrates the performance of the channel estimation, where the signal-to-noise ratio (SNR) values are given by

$$
\operatorname{SNR}=\frac{E\left(\left|h_{i, j}^{0}\right|^{2}\right)}{\operatorname{var}\left(h_{i, j}^{0}\right)}
$$

being $h_{i, j}^{0}$ the corresponding entry of $\mathbf{H}_{0}$, and $E()$ and $\operatorname{var}()$ being the sample expectation and variance, respectively. More details about the channel estimation algorithm and its performance can be found in [10].

Once $\mathbf{H}_{0}$ was estimated, the corresponding sequence of weight matrices, $\mathbf{W}[n]$, was calculated from (4). Each weight realization was fed to the weighting block and the overall channel realizations $\mathbf{H}_{e}[n]$ were estimated using again the method of [10]. These estimates are also depicted in Figure 4 but with square markers. The figure shows an excellent agreement between the sequence to emulate, $\mathbf{H}[n]$, and the actual emulated channels $\mathbf{H}_{e}[n]$. Similar agreement was observed for the rest of samples. 


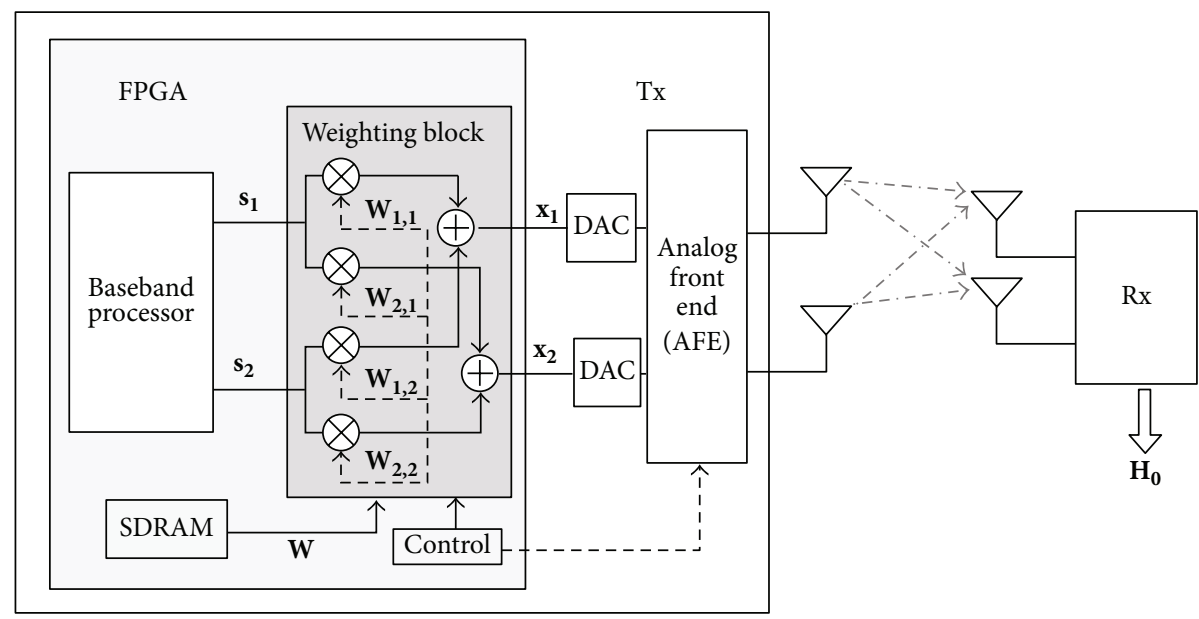

FIGURE 2: Scheme for $2 \times 2$ MIMO OTA testing and channel emulation.

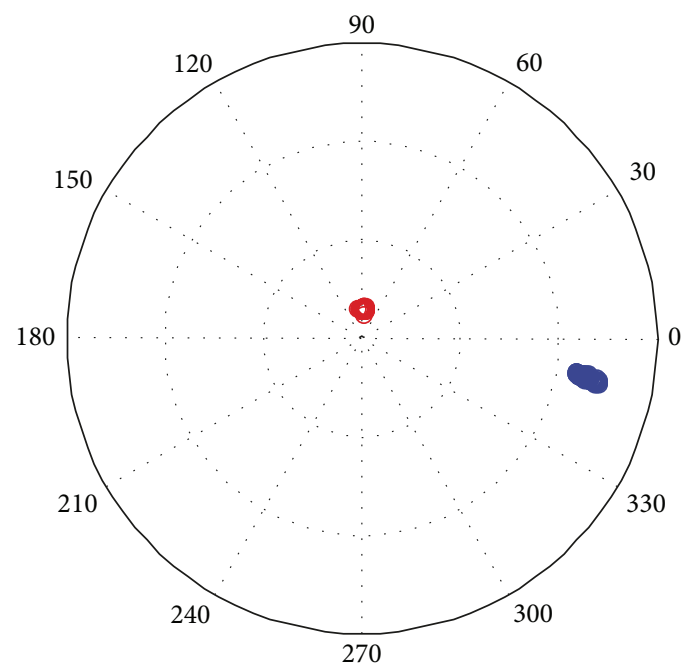

Tx antenna 1

Tx antenna 2

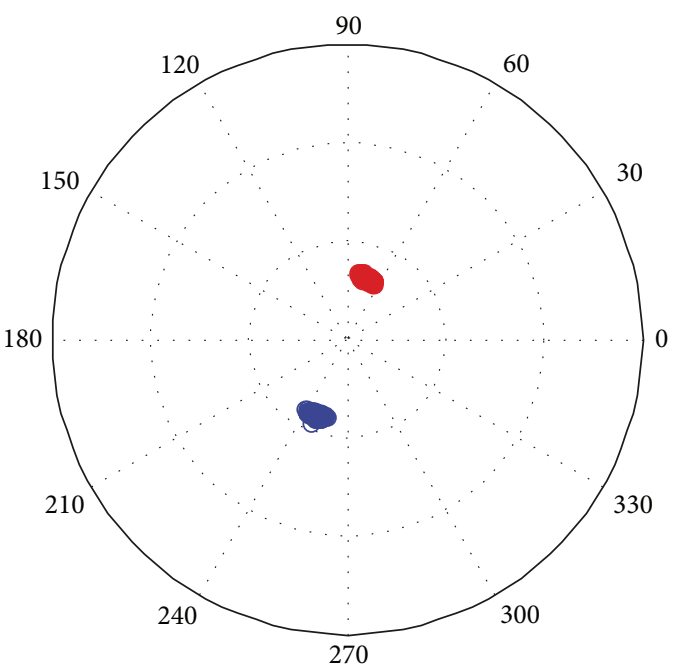

Tx antenna 1

Tx antenna 2

(a)

(b)

Figure 3: Estimates of $\mathbf{H}_{0}$. (a) Receive antenna 1. (b) Receive antenna 2.

TABle 2: Performance of the emulation scheme.

\begin{tabular}{lcccc}
\hline & $h_{11}$ & $h_{21}$ & $h_{12}$ & $h_{22}$ \\
\hline SNR (dB) & 31.2819 & 21.9089 & 22.7801 & 18.7281 \\
\hline
\end{tabular}

Table 2 shows the performance of the whole channel emulation procedure, where the SNR values are

$$
\operatorname{SNR}=\frac{E\left(\left|h_{i, j}\right|^{2}\right)}{E\left(\left|h_{i, j}-h_{i, j}^{e}\right|^{2}\right)}
$$

$h_{i, j}$ and $h_{i, j}^{e}$ being with the corresponding entries of $\mathbf{H}$ and $\mathbf{H}_{e}$, respectively. Comparing the values of Tables 1 and 2, one concludes that the emulation errors are mainly due to the errors in the estimation of $\mathbf{H}_{0}$.

\section{Conclusions}

This work proposes a simple and flexible method for narrowband MIMO OTA testing. It allows testing under any MIMO channel avoiding the need of channel emulators and other facilities like anechoic and reverberation chambers. The method is based on the linear combination of the baseband 


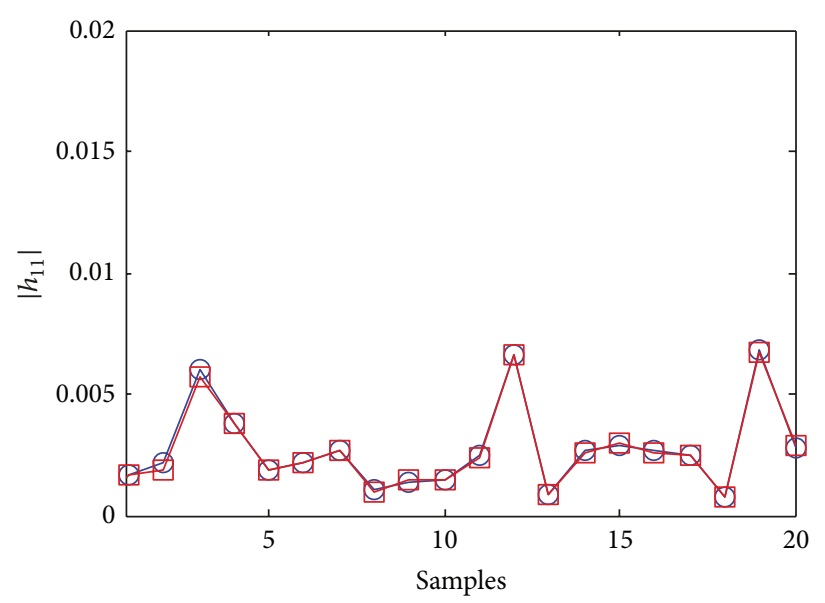

(a)

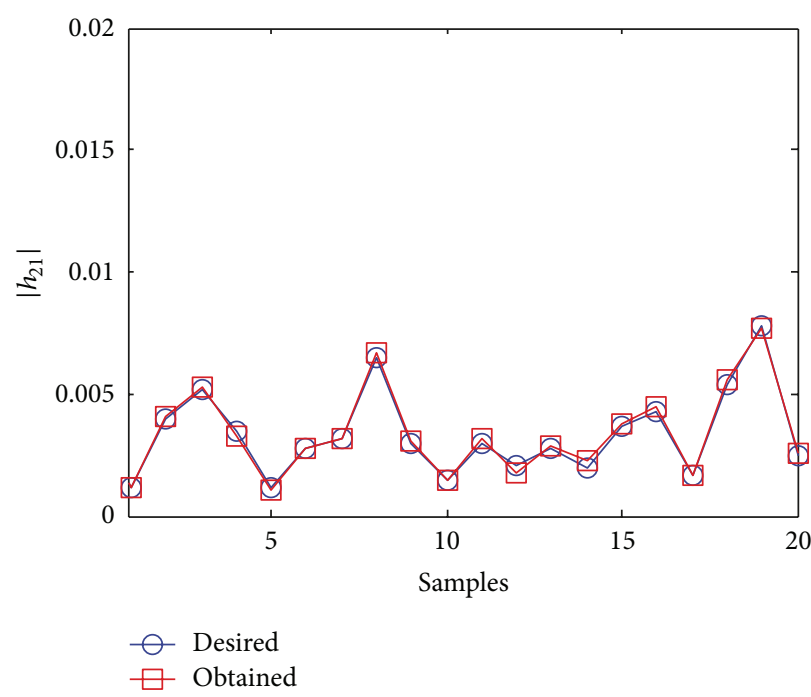

(c)

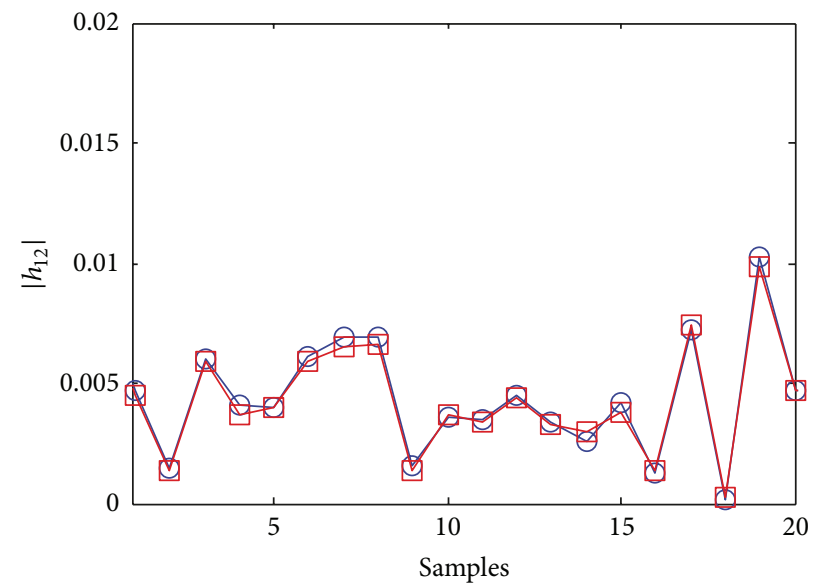

(b)

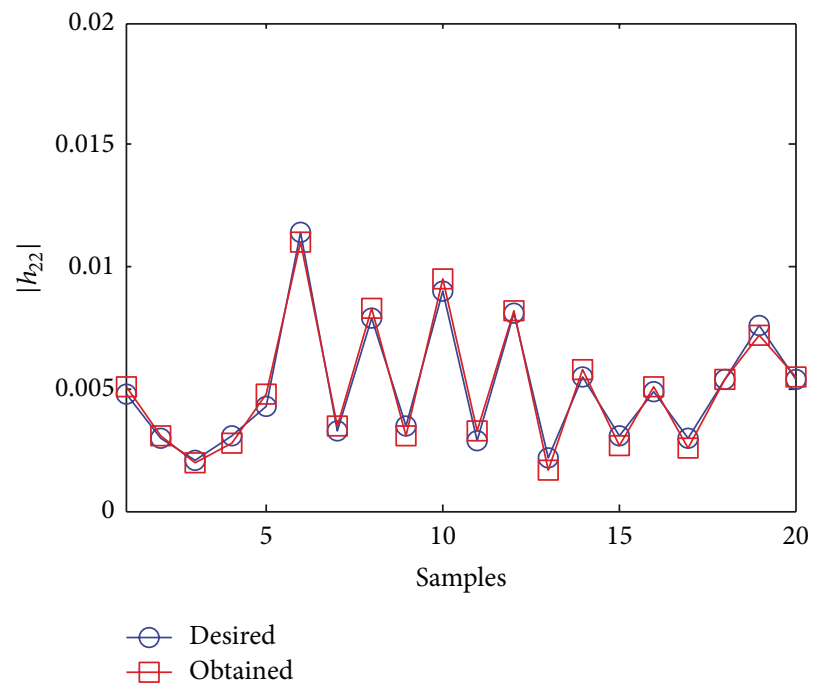

(d)

FIGURE 4: Comparison between the desired channel $\mathbf{H}[n]$ and the actual emulated channel $\mathbf{H}_{e}[n]$.

signals at the Tx. The solution is fully flexible in the sense that it can emulate any baseband equivalent channel realization by properly choosing the weights of the linear combination. These weights can be easily computed as functions of the channel to emulate and the actual channel between the Tx and $\mathrm{Rx}$. The method relies on the stationarity of such channel in the laboratory where the testbed is placed. In a preliminary stage, this channel must be estimated by the $\mathrm{BB}$ processor at the $\mathrm{Rx}$. In general, to emulate a $n_{T} \times n_{R}$ MIMO channel, a testbed with $n_{R} \times n_{R}$ antennas is required. The DUTs could be at the Tx and/or at the Rx. The DUT can be also a complete $\mathrm{Rx}$ provided that the $\mathrm{BB}$ processor at the $\mathrm{Rx}$ is able to estimate the equivalent baseband channel. The scheme is also well suited to test MIMO-specific algorithms (space-time coding, spatial multiplexing, etc.) that are usually implemented in the Tx and Rx baseband processors. To show its feasibility and performance, the proposed method was implemented on a conventional MIMO testbed with excellent results.

\section{Acknowledgments}

This work was supported by the Spanish Government, Ministerio de Ciencia e Innovacain (MICINN), under projects COSIMA (TEC2010-19545-C04-03) and COMONSENS (CSD2008-00010, CONSOLIDER-INGENIO 2010).

\section{References}

[1] L. Vielva, J. Vía, J. Gutiérrez, Ó. González, J. Ibáñez, and I. Santamaría, "Building a web platform for learning advanced digital communications using a MIMO testbed," in Proceedings of the IEEE International Conference on Acoustics, Speech and Signal Processing (ICASSP '10), Dallas, Tex, USA, 2010.

[2] S. Caban, C. Mehlführer, R. Langwieser, A. L. Scholtz, and M. Rupp, "Vienna MIMO testbed," Eurasip Journal on Applied Signal Processing, vol. 2006, Article ID 054868, 2006.

[3] A. Guillén i Fàbregas, M. Guillaud, D. T. M. Slock et al., "A MIMO-OFDM testbed for wireless local area networks," 
Eurasip Journal on Applied Signal Processing, vol. 2006, Article ID 18083, pp. 1-20, 2006.

[4] M. Rumney, R. Pirkl, M. H. Landmann, and D. A. SanchezHernandez, "MIMO over-the-air research, development, and testing," International Journal of Antennas and Propagation, vol. 2012, Article ID 467695, 8 pages, 2012.

[5] A. Kosako, M. Shinozawa, and Y. Karasawa, "Simplified configuration of fading emulator system for MIMO-OTA testing," in Proceedings of the 2nd International Conference on Wireless Communication, Vehicular Technology, Information Theory and Aerospace and Electronic Systems Technology, Wireless (VITAE '11), pp. 1-5, March 2011.

[6] Y. Karasawa, Y. Gunawan, S. Pasisngi, K. Nakada, and A. Kosako, "Development of a MIMO-OTA system with simplified configuration," Journal of the Korean Institute of Electromagnetic Engineering and Science, vol. 12, no. 1, pp. 77-84, 2012.

[7] J. D. Sanchez-Heredia, J. F. Valenzuela-Valdés, A. M. MartínezGonzález, and D. A. Sánchez-Hernández, "Emulation of MIMO rician-fading environments with mode-stirred reverberation chambers," IEEE Transactions on Antennas and Propagation, vol. 59, no. 2, pp. 654-660, 2011.

[8] M. García-Fernández, J. Sánchez-Heredia, A. MartínezGonzález, D. Sánchez-Hernández, and J. Valenzuela-Valdés, "Advances in mode-stirred reverberation chambers for wireless communication performance evaluation," IEEE Communications Magazine, vol. 49, no. 7, pp. 140-147, 2011.

[9] X. Chen, P. Kildal, C. Orlenius, and J. Carlsson, "Channel sounding of loaded reverberation chamber for over-the-air testing of wireless devices: coherence bandwidth versus average mode bandwidth and delay spread," IEEE Antennas and Wireless Propagation Letters, vol. 8, pp. 678-681, 2009.

[10] J. Gutiérrez, Ó. González, J. Pérez et al., "Frequency-domain methodology for measuring MIMO channels using a generic testbed," IEEE Transactions on Instrumentation and Measurement, vol. 60, no. 3, pp. 827-838, 2011.

[11] A. Paulraj, R. Nabar, and D. Gore, Introduction to Space-Time Wireless Communications, Cambridge University Press, 2003.

[12] M. Coldrey, "Modeling and capacity of polarized MIMO channels," in Proceedings of the IEEE 67th Vehicular Technology Conference (VTC '08), pp. 440-444, May 2008.

[13] T. Neubauer and P. C. F. Eggers, "Simultaneous characterization of polarization matrix components in pico cells," in Proceedings of the IEEE VTS 50th Vehicular Technology Conference (VTC '99), pp. 1361-1365, September 1999. 

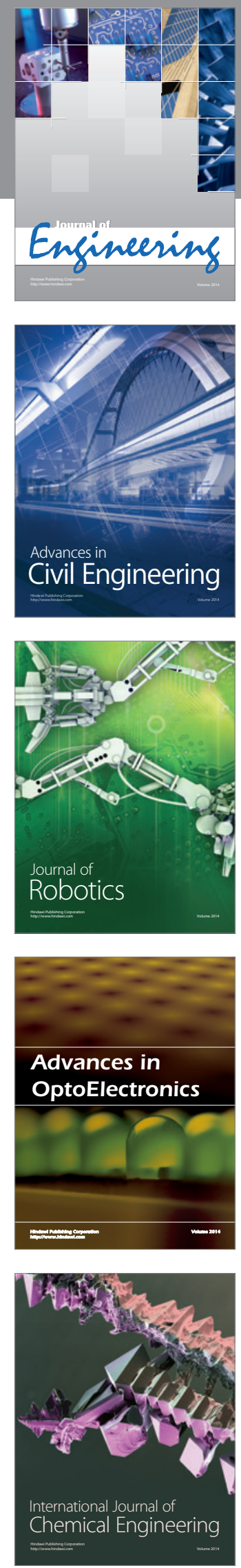

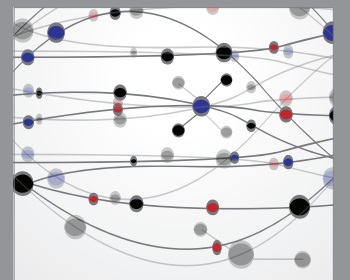

The Scientific World Journal
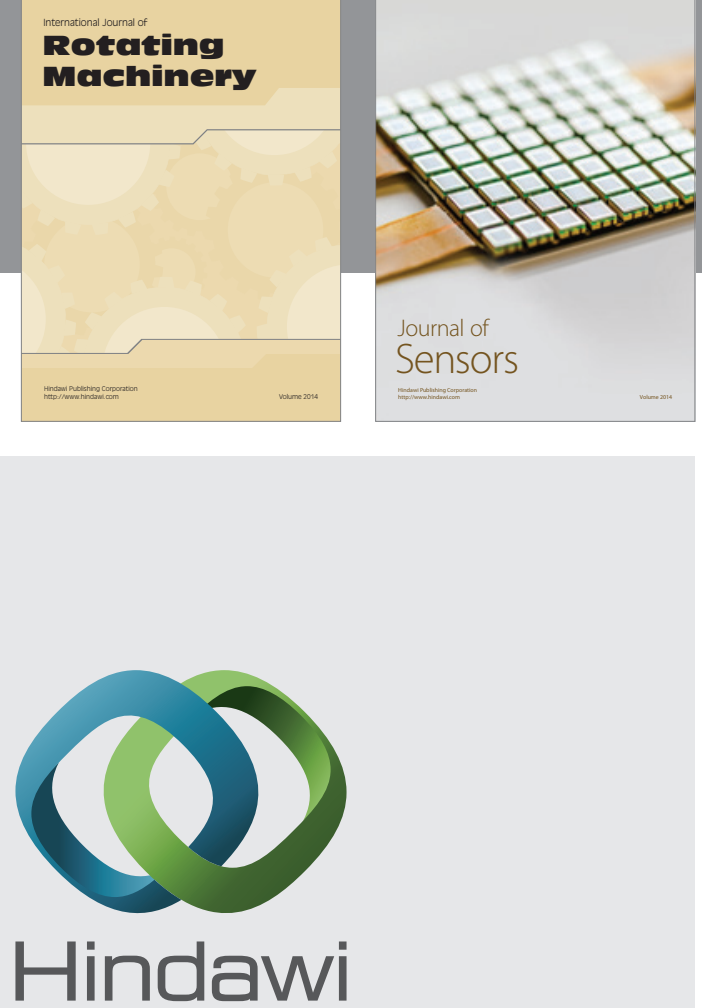

Submit your manuscripts at http://www.hindawi.com
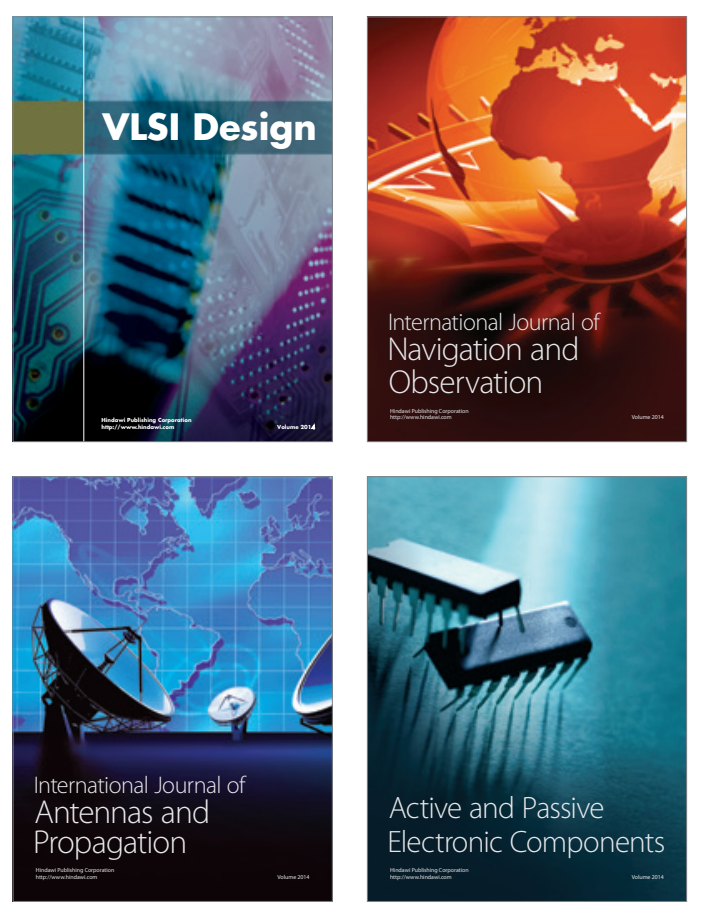
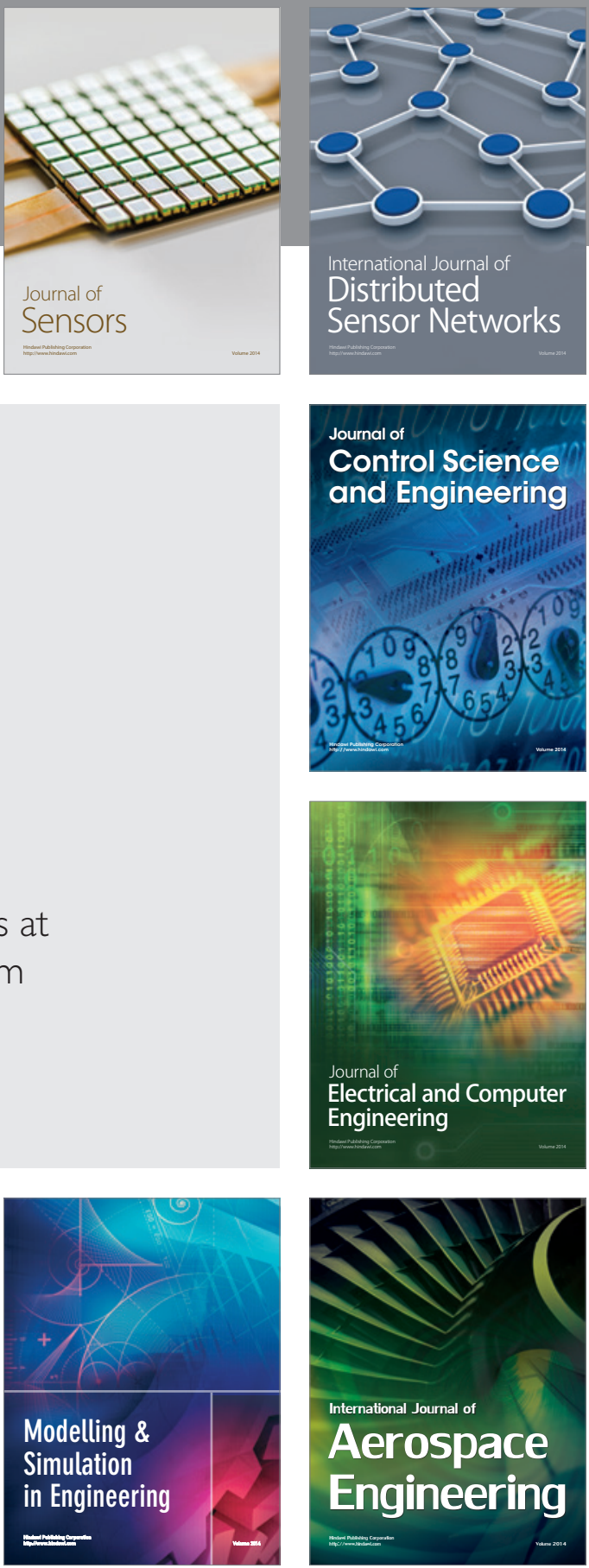

Journal of

Control Science

and Engineering
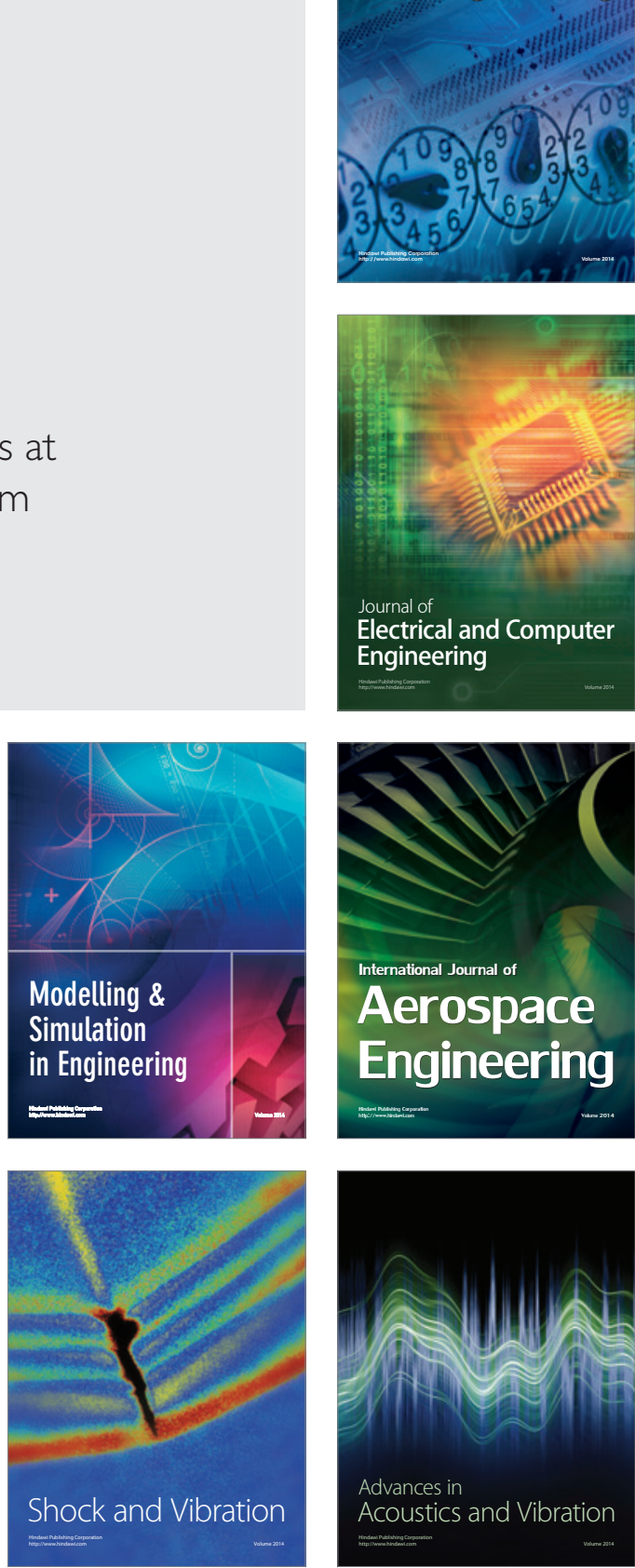